\title{
The 2-Periodic Interpolation Problem of Anti-Periodic Function
}

\author{
Ruiping Gao, Shangqin He, Cuiyi Xiao \& Huiquan Ma \\ School of Economics, Hebei Normal University of science and technology \\ 360 Hei Bei Street, Qinhuangdao, Hebei 066004, China \\ Tel: 86-137-8451-3076_E-mail: grp20081218@126.com
}

\begin{abstract}
By introducing the difference polynomial operator $p\left(\frac{1}{2 h} \Delta_{h}\right)$, a kind of 2-periodic $\left(0, P\left(\frac{1}{2 h} \Delta_{h}\right)\right)$ trigonometric Interpolation problem of anti-periodic function is discussed. Some conditions of existence of solutions are established and the explicit expressions are given under the corresponding conditions.
\end{abstract}

Keywords: Anti-periodic function, 2-periodic, Polynomial operator

The anti-periodic research on theoretical and applied mathematics have become one of the direction of mathematical research, has been widespread concern. The research, in the approximation and interpolation theory of function, about $\pi$ - cycle of $(0, m)$ trigonometric interpolation problem of anti-periodic function has attracted great attention and has been studied extensively after Delvos, FJ published their paper, see (Franz-Jurgen Delvos, Ludger Knoche. 1999)(Franz-Jurgen Delvos. 1993). In (Shangqin He, Xiangqian Hou. 2007), The author discussed that $\pi$ - cycle of 2-periodic $(0, m)$ trigonometric interpolation problem of anti-periodic function, and found the solution conditions for the existence, fundamental polynomials and interpolation polynomial of explicit expressions. However, in (Shangqin He, Xiangqian Hou. 2007), the theorem asked to be interpolation function has a m-order differentiable, not to be at the junction point interpolation function non-differentiable situation. By using differential operator instead of difference polynomial operator, we obtained the following conclusion, see (Shangqin He, Xiangqian Hou. 2006):

Theorem A: If $f(x)$ is a anti-periodic function with $\pi_{\text {- cycle and }} P\left(i \frac{\sin (k+n) h}{h}\right)+P\left(i \frac{\sin (k-n) h}{h}\right) \neq 0$, then we can get $Q_{n}^{P\left(\frac{1}{2 h} \Delta_{h}\right)} f(x) \in \omega_{2 n-1}^{\perp}$ is uniquely given by

$Q_{n}^{P\left(\frac{1}{2 h} \Delta_{h}\right)} f(x)=\sum_{k=0}^{n-1} f\left(x_{k}\right) G\left(x-x_{k}\right) \sum_{k=0}^{n-1}\left(P\left(\frac{1}{2 h} \Delta_{h}\right) f\right)\left(y_{k}\right) H\left(x-x_{k}\right)$

in which

$Q_{n}^{P\left(\frac{1}{2 h} \Delta_{h}\right)} f\left(x_{k}\right)=f\left(x_{k}\right),\left(P\left(\frac{1}{2 h} \Delta_{h}\right) Q_{n}^{P\left(\frac{1}{2 h} \Delta_{h}\right)} f\right)\left(y_{k}\right)=\left(P\left(\frac{1}{2 h} \Delta_{h}\right) f\right)\left(y_{k}\right)$.

There is some discussion about the Construction of $G(x)$ and $H(x)$ in the theorem A described by (Shangqin He, Xiangqian Hou. 2006), but they haven't get the fundamental polynomials and interpolation polynomial of explicit expressions. In this paper, we refer to discuss a kind of 2-periodic $\left(0, P\left(\frac{1}{2 h} \Delta_{h}\right)\right)$ trigonometric Interpolation problem of anti-periodic function based on (Shangqin He, Xiangqian Hou. 2007). The main problems are following:

For any equidistant nodes $x_{m}=\frac{k \pi}{n}, y_{m}=x_{m}+\frac{\pi}{2 n}(m=0,1,2, \cdots, n-1)$ and two sets of complex $\left\{\alpha_{m}\right\}_{m=0}^{n-1},\left\{\beta_{m}\right\}_{m=0}^{n-1}$, there exists only $T(x) \in \omega_{2 n-1}^{\perp}$ under which conditions such that

$T\left(x_{m}\right)=\alpha_{m},\left(P\left(\frac{1}{2 h} \Delta_{h}\right) T\right)\left(y_{m}\right)=\beta_{m}$

Assume $\tau_{n}$ is the trigonometric polynomials space which maximum number is not exceeding n, thus,

$\tau_{0}=\operatorname{span}\{1\}, \tau_{k}=\tau_{k-1}+\operatorname{span}\{\cos k x, \sin k x\} \omega_{n}=\left\{T \in \tau_{n} \mid T(x+\pi)=T(x)\right\}$

If $\omega_{n}=\left\{T \in \tau_{n} \mid T(x+\pi)=T(x)\right\}$ is the trigonometric polynomials subspace with $\pi$-cycle, thus, we can 
conclude that

$$
\omega_{0}=\tau_{0}, \omega_{2 k+1}=\omega_{2 k}, \omega_{2 k+2}=\omega_{2 k+1}+\operatorname{span}\{\cos 2(k+1) x, \sin 2(k+1) x\} .
$$

Suppose $\omega_{n}^{\perp}=\left\{T \in \tau_{n} \mid T(x+\pi)=-T(x)\right\}$ is the anti- periodic trigonometric polynomials subspace with $\pi$-cycle, then

$\omega_{0}^{\perp}=\{0\}, \omega_{2 k+2}^{\perp}=\omega_{2 k+1}^{\perp}, \omega_{2 k+1}^{\perp}=\omega_{2 k}^{\perp}+\operatorname{span}\{\cos (2 k+1) x, \sin (2 k+1) x\}$.

Obviously, $\tau_{n}=\omega_{n} \oplus \omega_{\frac{1}{n}}$, for any $f(x) \in C_{2 \pi}$, if $0<h<\frac{\pi}{2 n}, m \in N$, define difference operator $\Delta$ :

$$
\begin{aligned}
& \Delta_{h}^{0} f(x)=f(x), \Delta_{h} f(x)=\Delta_{h}^{1} f(x)=f(x+h)-f(x-h), \\
& \Delta_{h}^{m} f(x)=\Delta\left[\Delta_{h}^{m-1} f(x)\right]=\sum_{k=0}^{m}(-1)^{k}\left(\begin{array}{l}
m \\
k
\end{array}\right) f[x+(m-2 k) h] \quad(m \geq 2) .
\end{aligned}
$$

Set $P(t)=\sum_{j=1}^{M} p_{j} t^{j}(p \in R) \quad$ is real algebraic polynomial, $M \in Z^{+}, \quad P(D)=\sum_{j=1}^{M} P_{j} D^{j}\left(D=\frac{d}{d x}\right)$ is the differential polynomials operator exported by $P(t)$ and $P\left(\frac{1}{2 h} \Delta_{h}\right)=\sum_{j=1}^{M} P_{j} \frac{1}{(2 h)^{j}} \Delta_{h}^{j}$ is the difference polynomials operator exported by $P(t)$.

The main result is the following:

Theorem 1 (I) Suppose $P(t)$ is real even polynomial, there exists a unique trigonometric polynomial ${ }^{T(x)}$, such that

$T\left(x_{m}\right)=\alpha_{m},\left(P\left(\frac{1}{2 h} \Delta_{h}\right) T\right)\left(y_{m}\right)=\beta_{m} ;$

Where $P\left(\frac{i \sin (2 k-1) h}{h}\right)+P\left(\frac{i \sin (2 n-2 k+1) h}{h}\right) \neq 0$.

(II) Suppose $P(t)$ is real odd polynomial, there exists a unique trigonometric polynomial ${ }^{T(x)}$, such that $T\left(x_{m}\right)=\alpha_{m}, \quad\left(P\left(\frac{1}{2 h} \Delta_{h}\right) T\right)\left(y_{m}\right)=\beta_{m}$.

Where $P\left(\frac{i \sin (2 k-1) h}{h}\right)-P\left(\frac{i \sin (2 n-2 k+1) h}{h}\right) \neq 0$.

Assume $r_{m}(x), \rho_{m}(x)$ denote the considered problem of fundamental polynomials, respectively. Then $r_{m}(x)=r_{0}\left(x-x_{m}\right), \rho_{m}(x)=\rho_{0}\left(x-x_{m}\right), m=1,2, \cdots, n-1$

Where $r_{0}(x), \rho_{0}(x)$ satisfy $r_{0}\left(x_{m}\right)=\delta_{0 m}, \quad\left(\left(P\left(\frac{1}{2 h} \Delta_{h}\right) r_{0}\right)\left(y_{m}\right)=0\right.$

$$
\rho_{0}\left(x_{m}\right)=0, \quad\left(\left(P\left(\frac{1}{2 h} \Delta_{h}\right) \rho_{0}\right)\left(y_{m}\right)=\delta_{0 m}, m=0,1,2, \cdots, n-1\right.
$$

Theorem 2 ( I ) Suppose $P(t)$ is the real even polynomial,

$$
\begin{aligned}
& \text { if } P\left(\frac{i \sin (2 k-1) h}{h}\right)+P\left(\frac{i \sin (2 n-2 k+1) h}{h}\right) \neq 0 \text {, then } \\
& r_{0}(x)=\sum_{k=1}^{n} \frac{2 P\left(\frac{i \sin (2 n-2 k+1) h}{h}\right)}{n\left[P\left(\frac{i \sin (2 k-1) h}{h}\right)+P\left(\frac{i \sin (2 n-2 k+1) h}{h}\right)\right]} \cos (2 k-1) x
\end{aligned}
$$


$\rho_{0}(x)=\sum_{k=1}^{n} \frac{2}{n\left[P\left(\frac{i \sin (2 k-1) h}{h}\right)+P\left(\frac{i \sin (2 n-2 k+1) h}{h}\right)\right]} \sin (2 k-1) x$

(II) Suppose $P(t)$ is the real odd polynomial,

$$
\text { if } \begin{aligned}
P\left(\frac{i \sin (2 k-1) h}{h}\right)-P\left(\frac{i \sin (2 n-2 k+1) h}{h}\right) \neq 0 \text {, then } \\
r_{0}(x)=-\sum_{k=1}^{n} \frac{2 P\left(\frac{i \sin (2 n-2 k+1) h}{h}\right)}{n\left[P\left(\frac{i \sin (2 k-1) h}{h}\right)-P\left(\frac{i \sin (2 n-2 k+1) h}{h}\right)\right]} \cos (2 k-1) x \\
\rho_{0}(x)=\sum_{k=1}^{n} \frac{2}{n\left[P\left(\frac{i \sin (2 k-1) h}{h}\right)-P\left(\frac{i \sin (2 n-2 k+1) h}{h}\right)\right]} \sin (2 k-1) x
\end{aligned}
$$

Theorem 3 ( I ) Suppose $f(x)$ is anti-periodic function with $\pi$-cycle, $P(t)$ is real even polynomial, if $P\left(\frac{i \sin (2 k-1) h}{h}\right)+P\left(\frac{i \sin (2 n-2 k+1) h}{h}\right) \neq 0$, there exists a unique trigonometric polynomial $T(x) \in \omega_{2 n-1}^{\perp}$ satisfied $T(x)=\sum_{m=0}^{n-1} f\left(x_{m}\right) r_{0}\left(x-x_{m}\right)+\sum_{m=0}^{n-1}\left(P\left(\frac{1}{2 h} \Delta_{h}\right) f\right)\left(y_{m}\right) \rho_{0}\left(x-x_{m}\right)$

such that $T\left(x_{m}\right)=f\left(x_{m}\right),\left(P\left(\frac{1}{2 h} \Delta_{h}\right) T\right)\left(y_{m}\right)=\left(P\left(\frac{1}{2 h} \Delta_{h}\right) f\right)\left(y_{m}\right)$.

Where $r_{0}(x)$ and $\rho_{0}(x)$ is given by (1) and (2).

(II) Suppose $f(x)$ is anti-periodic function with $\pi$-cycle, $P(t)$ is real odd polynomial, if $P\left(\frac{i \sin (2 k-1) h}{h}\right)-P\left(\frac{i \sin (2 n-2 k+1) h}{h}\right) \neq 0$, there exists a unique trigonometric polynomial $T(x) \in \omega_{2 n-1}^{\perp}$ satisfied

$T(x)=\sum_{m=0}^{n-1} f\left(x_{m}\right) r_{0}\left(x-x_{m}\right)+\sum_{m=0}^{n-1}\left(P\left(\frac{1}{2 h} \Delta_{h}\right) f\right)\left(y_{m}\right) \rho_{0}\left(x-x_{m}\right)$

such that $\quad T\left(x_{m}\right)=f\left(x_{m}\right),\left(P\left(\frac{1}{2 h} \Delta_{h}\right) T\right)\left(y_{m}\right)=\left(P\left(\frac{1}{2 h} \Delta_{h}\right) f\right)\left(y_{m}\right)$,

Where $r_{0}(x)$ and $\rho_{0}(x)$ is given by (3) and (4).

\section{Lemma}

Lemma 1 (Shangqin He, Xiangqian Hou. 2007) If $T(x) \in \omega_{2 n-1}^{\perp}, \quad$ there exists constant $a_{j}, b_{j}$ $(j=1,3, \cdots, 2 n-1)$, then

$T(x)=\sum_{j=1}^{n}\left(a_{2 j-1} \cos (2 j-1) x+b_{2 j-1} \sin (2 j-1) x\right)$

Lemma 2(Shangqin He, Xiangqian Hou. 2007) If

$$
T(x)=\sum_{j=1}^{n}\left(a_{2 j-1} \cos (2 j-1) x+b_{2 j-1} \sin (2 j-1) x\right)
$$

$T\left(x_{m}\right)=\frac{1}{2} \sum_{j=1}^{n}\left\{\left(a_{2 j-1}+a_{2 n-(2 j-1)}\right) \cos (2 j-1) x_{m}+\left(b_{2 j-1}-b_{2 n-(2 j-1)}\right) \sin (2 j-1) x_{m}\right\}$

Lemma 3 (1) Suppose $j$ is odd, $\Delta_{h}^{j} \cos (2 k-1) x=(2 i \sin (2 k-1) h)^{j} \cos (2 k-1) x$, 


$$
\Delta_{h}^{j} \sin (2 k-1) x=(2 i \sin (2 k-1) h)^{j} \sin (2 k-1) x
$$

(2) Suppose $j$ is even,

$$
\begin{aligned}
& \Delta_{h}^{j} \cos (2 k-1) x=i(2 i \sin (2 k-1) h)^{j} \sin (2 k-1) x, \\
& \Delta_{h}^{j} \sin (2 k-1) x=-i(2 i \sin (2 k-1) h)^{j} \cos (2 k-1) x .
\end{aligned}
$$

Proof: By Induction obviously,

Suppose $j$ is odd, $\Delta_{h}^{j} \cos (2 k-1) x=(2 i \sin (2 k-1) h)^{j} \cos (2 k-1) x$,

$$
\Delta_{h}^{j} \sin (2 k-1) x=(2 i \sin (2 k-1) h)^{j} \sin (2 k-1) x ;
$$

Suppose $j$ is even, $\Delta_{h}^{j} \cos (2 k-1) x=i(2 i \sin (2 k-1) h)^{j} \sin (2 k-1) x$,

$$
\Delta_{h}^{j} \sin (2 k-1) x=-i(2 i \sin (2 k-1) h)^{j} \cos (2 k-1) x .
$$

Lemma 4(Shangqin He, Xiangqian Hou. 2007) Assume

$$
T(x)=\sum_{j=1}^{n}\left(a_{2 j-1} \cos (2 j-1) x+b_{2 j-1} \sin (2 j-1) x\right),
$$
then

(1) $T\left(x_{m}\right)=\delta_{0 m}(m=0,1,2, \cdots, n-1)$ if and only if

$$
a_{2 j-1}+a_{2 n-(2 j-1)}=\frac{2}{n}, \quad b_{2 j-1}-b_{2 n-(2 j-1)}=0, j=1,2, \cdots, n, \quad \begin{gathered}
\delta_{0 m} \\
=
\end{gathered}=\left\{\begin{array}{l}
1, m=0 \\
0, m \neq 0
\end{array}\right.
$$

(2) $T\left(x_{m}\right)=0(m=0,1,2, \cdots, n-1)$ if and only if

$$
a_{2 j-1}+a_{2 n-(2 j-1)}=0 \quad b_{2 j-1}-b_{2 n-(2 j-1)}=0, j=1,2, \cdots, n \text {, }
$$

for any $n \in N$,

Lemma 5 (Shangqin He, Xiangqian Hou. 2007) Assume

$$
T(x)=\sum_{j=1}^{n}\left(a_{2 j-1} \cos (2 j-1) x+b_{2 j-1} \sin (2 j-1) x\right)
$$
then

(1) $T\left(y_{m}\right)=\delta_{0 m}(m=0,1,2, \cdots, n-1)$ if and only if

$a_{2 j-1}-a_{2 n-(2 j-1)}=\frac{2}{n} \cos (2 j-1) y_{0} \quad b_{2 j-1}+b_{2 n-(2 j-1)}=\frac{2}{n} \sin (2 j-1) y_{0}, j=1,2, \cdots, n$

(2) $T\left(y_{m}\right)=0(m=0,1,2, \cdots, n-1)$ if and only if

$a_{2 j-1}+a_{2 n-(2 j-1)}=0 \quad, \quad b_{2 j-1}-b_{2 n-(2 j-1)}=0, j=1,2, \cdots, n$

for any $n \in N$.

\section{The proof of theorem}

Suppose $T(x)=\sum_{k=1}^{n}\left[a_{2 k-1} \cos (2 k-1) x+b_{2 k-1} \sin (2 k-1) x\right]$,

Since $y_{m}=x_{m}+\frac{\pi}{2 n}$ and $y_{0}=\frac{\pi}{2 n}$, we can conclude that 
$T\left(y_{m}\right)=T\left(x_{m}+y_{0}\right)=\sum_{k=1}^{n}\left[A_{2 k-1} \cos (2 k-1) x_{m}+B_{2 k-1} \sin (2 k-1) x_{m}\right]$,

in which,

$$
\begin{aligned}
A_{2 k-1}=a_{2 j-1} \cos (2 j-1) y_{0}+b_{2 j-1} \sin (2 j-1) y_{0} \\
\quad B_{2 k-1}=b_{2 j-1} \cos (2 j-1) y_{0}-a_{2 j-1} \sin (2 j-1) y_{0}
\end{aligned}
$$

\subsection{The proof of theorem.}

Proof: If $P(t)$ is the real even polynomial, define

$U_{2 n-1}(x)=\sum_{k=1}^{n}\left[A_{2 k-1} \cos (2 k-1) x+B_{2 k-1} \sin (2 k-1) x\right]$

then, $P\left(\frac{1}{2 h} \Delta_{h}\right) T\left(y_{m}\right)=P\left(\frac{1}{2 h} \Delta_{h}\right) U_{2 n-1}\left(x_{m}\right)$

$P\left(\frac{1}{2 h} \Delta_{h}\right) U_{2 n-1}(x) \sum_{j=1}^{M} P_{j}\left(\frac{1}{2 h}\right)^{j} \Delta_{h}^{j} \sum_{k=1}^{n}\left[A_{2 k-1} \cos (2 k-1) x+B_{2 k-1} \sin (2 k-1) x\right]$

$\sum_{j=1}^{M} \sum_{k=1}^{n} P_{j}\left(\frac{1}{2 h}\right)^{j} \Delta_{h}^{j}\left[A_{2 k-1} \cos (2 k-1) x+B_{2 k-1} \sin (2 k-1) x\right]$

Applying the lemma 3,

$P\left(\frac{1}{2 h} \Delta_{h}\right) U_{2 n-1}(x)$

$=\sum_{j=1}^{M} P_{j}\left(\frac{1}{2 h}\right)^{j} \sum_{k=1}^{n}\left[(2 i)^{j} \sin ^{j}(2 k-1) h \cdot A_{2 k-1} \cos (2 k-1) x+(2 i)^{j} \sin ^{j}(2 k-1) h \cdot B_{2 k-1} \sin (2 k-1) x\right]$

$=\sum_{k=1}^{n}\left[P\left(\frac{i \sin (2 k-1) h}{h}\right) \cdot\left(A_{2 k-1} \cos (2 k-1) x+B_{2 k-1} \sin (2 k-1) x\right)\right]$.

By the lemma 2,

$P\left(\frac{1}{2 h} \Delta_{h}\right) U_{2 n-1}\left(x_{m}\right)$

$=\frac{1}{2} \sum_{k=1}^{n}\left\{\left[P\left(\frac{i \sin (2 k-1) h}{h}\right) A_{2 k-1}+P\left(\frac{i \sin (2 n-2 k+1) h}{h}\right) A_{2 n-2 k+1}\right] \cos (2 k-1) x_{m}+\right.$

$\left[P\left(\frac{i \sin (2 k-1) h}{h}\right) B_{2 k-1}-P\left(\frac{i \sin (2 n-2 k+1) h}{h}\right) B_{2 n-2 k+1}\right] \sin (2 k-1) x_{m}$.

Since $T\left(x_{m}\right)=0, P\left(\frac{1}{2 h} \Delta_{h}\right) U_{2 n-1}\left(x_{m}\right)=0$, in view of lemma 4, we can conclude that

$\left\{\begin{array}{l}a_{2 k-1}+a_{2 n-2 k+1}=0 \\ b_{2 k-1}-b_{2 n-2 k+1}=0\end{array}\right.$

and

$\left\{\begin{array}{l}P\left(\frac{i \sin (2 k-1) h}{h}\right) A_{2 k-1}+P\left(\frac{i \sin (2 n-2 k+1) h}{h}\right) A_{2 n-2 k+1}=0 \\ P\left(\frac{i \sin (2 k-1) h}{h}\right) B_{2 k-1}-P\left(\frac{i \sin (2 n-2 k+1) h}{h}\right) B_{2 n-2 k+1}=0\end{array}\right.$ 
It follows from (5), (6) and (7), we can find that $\left\{\begin{array}{l}\left(P\left(\frac{i \sin (2 k-1) h}{h}\right)+P\left(\frac{i \sin (2 n-2 k+1) h}{h}\right) \cos (2 k-1) y_{0} a_{2 k-1}+\right. \\ \left(P\left(\frac{i \sin (2 k-1) h}{h}\right)+P\left(\frac{i \sin (2 n-2 k+1) h}{h}\right) \sin (2 k-1) y_{0} b_{2 k-1}=0\right. \\ \left(P\left(\frac{i \sin (2 k-1) h}{h}\right)+P\left(\frac{i \sin (2 n-2 k+1) h}{h}\right) \cos (2 k-1) y_{0} b_{2 k-1}-\right. \\ \left(P\left(\frac{i \sin (2 k-1) h}{h}\right)+P\left(\frac{i \sin (2 n-2 k+1) h}{h}\right) \sin (2 k-1) y_{0} a_{2 k-1}=0\right.\end{array}\right.$.

Obviously, when $P\left(\frac{i \sin (2 k-1) h}{h}\right)+P\left(\frac{i \sin (2 n-2 k+1) h}{h}\right) \neq 0$ equations(Herui Zhang, Bingxin Hao. 1979). is hold, there exist solutions to the In the same way, we can conclude the results of (II). The proof is complete.

\subsection{The proof of theorem 2.}

Proof: Suppose $P(t)$ is the real even polynomial,

$P\left(\frac{1}{2 h} \Delta_{h}\right) r_{0}\left(y_{m}\right)=\frac{1}{2} \sum_{k=1}^{n}\left\{P\left(\frac{i \sin (2 k-1) h}{h}\right) A_{2 k-1}+P\left(\frac{i \sin (2 n-2 k+1) h}{h}\right) A_{2 n-2 k+1}\right] \cos (2 k-1) x_{m}+$

$\left.\left.P\left(\frac{i \sin (2 k-1) h}{h}\right) B_{2 k-1}-P\left(\frac{i \sin (2 n-2 k+1) h}{h}\right) B_{2 n-2 k+1}\right] \sin (2 k-1) x_{m}\right\}$,

Since $r_{0}\left(x_{m}\right)=\delta_{0 m}$ and $P\left(\frac{1}{2 h} \Delta_{h}\right) r_{0}\left(y_{m}\right)=0$ are hold, applying the lemma 4,we can find that $\left\{\begin{array}{l}a_{2 k-1}+a_{2 n-2 k+1}=\frac{2}{n} \\ b_{2 k-1}-b_{2 n-2 k+1}=0\end{array}\right.$, and in view of lemma 5 , we can find that

$$
\left\{\begin{array}{l}
P\left(\frac{i \sin (2 k-1) h}{h}\right) A_{2 k-1}+P\left(\frac{i \sin (2 n-2 k+1) h}{h}\right) A_{2 n-2 k+1}=0 \\
P\left(\frac{i \sin (2 k-1) h}{h}\right) B_{2 k-1}-P\left(\frac{i \sin (2 n-2 k+1) h}{h}\right) B_{2 n-2 k+1}=0
\end{array}\right.
$$

It follows from (5), (6) and (8), we can find that

$$
\left\{\begin{array}{l}
\left(P\left(\frac{i \sin (2 k-1) h}{h}\right)+P\left(\frac{i \sin (2 n-2 k+1) h}{h}\right)\right) \cos (2 k-1) y_{0} a_{2 k-1}+ \\
\left(P\left(\frac{i \sin (2 k-1) h}{h}\right)+P\left(\frac{i \sin (2 n-2 k+1) h}{h}\right)\right) \sin (2 k-1) y_{0} b_{2 k-1}=\frac{2}{n} P\left(\frac{i \sin (2 n-2 k+1) h}{h}\right) \cos (2 k-1) y_{0} \\
\left(P\left(\frac{i \sin (2 k-1) h}{h}\right)+P\left(\frac{i \sin (2 n-2 k+1) h}{h}\right)\right) \cos (2 k-1) y_{0} b_{2 k-1}- \\
\left(P\left(\frac{i \sin (2 k-1) h}{h}\right)+P\left(\frac{i \sin (2 n-2 k+1) h}{h}\right)\right) \sin (2 k-1) y_{0} a_{2 k-1}=-\frac{2}{n} P\left(\frac{i \sin (2 n-2 k+1) h}{h}\right) \sin (2 k-1) y_{0}
\end{array}\right.
$$

From this, we can calculate that

$$
a_{2 k-1}=\frac{2 P\left(\frac{i \sin (2 n-2 k+1) h}{h}\right)}{n\left(P\left(\frac{i \sin (2 k-1) h}{h}\right)+P\left(\frac{i \sin (2 n-2 k+1) h}{h}\right)\right)}, b_{2 k-1}=0,
$$


thus,

$$
r_{0}(x)=\sum_{k=1}^{n} \frac{2 P\left(\frac{i \sin (2 n-2 k+1) h}{h}\right)}{n\left[P\left(\frac{i \sin (2 k-1) h}{h}\right)+P\left(\frac{i \sin (2 n-2 k+1) h}{h}\right)\right]} \cos (2 k-1) x
$$

In the same way, we can conclude that $P\left(\frac{1}{2 h} \Delta_{h}\right) \rho_{0}\left(y_{m}\right)$

$$
\begin{aligned}
& \quad \frac{1}{2} \sum_{k=1}^{n}\left\{\left[P\left(\frac{i \sin (2 k-1) h}{h}\right) A_{2 k-1}+P\left(\frac{i \sin (2 n-2 k+1) h}{h}\right) A_{2 n-2 k+1}\right] \cos (2 k-1) x_{m}+\right. \\
& \left.\left[P\left(\frac{i \sin (2 k-1) h}{h}\right) B_{2 k-1}-P\left(\frac{i \sin (2 n-2 k+1) h}{h}\right) B_{2 n-2 k+1}\right] \sin (2 k-1) x_{m}\right\},
\end{aligned}
$$

in which, $\rho_{0}\left(x_{m}\right)=0$ and $P\left(\frac{1}{2 h} \Delta_{h}\right) \rho_{0}\left(y_{m}\right)=\delta_{0 m}$.

By lemma 4, we can find that $\left\{\begin{array}{l}a_{2 k-1}+a_{2 n-2 k+1}=0 \\ b_{2 k-1}-b_{2 n-2 k+1}=0\end{array}\right.$,

in view of lemma 5 , we obtain that

$$
\left\{\begin{array}{l}
P\left(\frac{i \sin (2 k-1) h}{h}\right) A_{2 k-1}+P\left(\frac{i \sin (2 n-2 k+1) h}{h}\right) A_{2 n-2 k+1}=\frac{2}{n} \cos (2 k-1) y_{0} \\
P\left(\frac{i \sin (2 k-1) h}{h}\right) B_{2 k-1}-P\left(\frac{i \sin (2 n-2 k+1) h}{h}\right) B_{2 n-2 k+1}=\frac{2}{n} \sin (2 k-1) y_{0}
\end{array}\right.
$$

In view of (5), (6) and (9), we can conclude that

$$
\left\{\begin{array}{l}
\left(P\left(\frac{i \sin (2 k-1) h}{h}\right)+P\left(\frac{i \sin (2 k-1) h}{h}\right)\right) \cos (2 k-1) y_{0} a_{2 k-1}+ \\
\left(P\left(\frac{i \sin (2 k-1) h}{h}\right)+P\left(\frac{i \sin (2 k-1) h}{h}\right)\right) \sin (2 k-1) y_{0} b_{2 k-1}=\frac{2}{n} \cos (2 k-1) y_{0} \\
\left(P\left(\frac{i \sin (2 k-1) h}{h}\right)+P\left(\frac{i \sin (2 k-1) h}{h}\right)\right) \cos (2 k-1) y_{0} b_{2 k-1}- \\
\left(P\left(\frac{i \sin (2 k-1) h}{h}\right)+P\left(\frac{i \sin (2 k-1) h}{h}\right)\right) \sin (2 k-1) y_{0} a_{2 k-1}=\frac{2}{n} \sin (2 k-1) y_{0}
\end{array}\right.
$$

From this, we can calculate that $a_{2 k-1}=0$,

$$
\begin{aligned}
& b_{2 k-1}=\frac{2}{n\left[P\left(\frac{i \sin (2 k-1) h}{h}\right)+P\left(\frac{i \sin (2 n-2 k+1) h}{h}\right)\right]} \\
& \rho_{0}(x)=\sum_{k=1}^{n} \frac{2}{n\left[P\left(\frac{i \sin (2 k-1) h}{h}\right)+P\left(\frac{i \sin (2 n-2 k+1) h}{h}\right)\right]} \sin (2 k-1) x \\
& \text { Thus, }
\end{aligned}
$$

In the same way, we can conclude the results of (II). The proof is complete.

2.3 The proof of theorem 3.

By virtue of Theorem 1 and Theorem, we can conclude the results. The proof is complete.

\section{References}

Franz-Jurgen Delvos, Ludger Knoche. (1999). Lacunary interpolation by antiperiodic trigonometric polynomials. BIT, 1999, 39(3): 430-450. 
Franz-Jurgen Delvos. (19993). Hermite Interpolation with trigonometric polynomials. BIT, 1993, 33(1):113-123. Herui Zhang, Bingxin Hao. (1979). Advanced Algebra. Beijing: High Education Press.

Shangqin He, Xiangqian Hou. (2006). The $\left(0, P\left(\frac{1}{2 h} \Delta_{h}\right)\right)$ trigonometric interpolations of antiperiodic functions. Journal of Sichuan Normal University(Natural Science), 2006, 29(05): 573-576.

Shangqin He, Xiangqian Hou. (2007). On the 2-periodic $(0, \mathrm{~m})$ trigonometric interpolation of antiperiodic function. Pure and Applied Mathematics, 2007, 23(04): 513-518. 